\title{
28 Research Suare \\ Multicolor Multifocal 3D Microscopy Using In-situ Optimization of A Spatial Light Modulator
}

\author{
M. Junaid Amin \\ Princeton University \\ Tian Zhao \\ Princeton University \\ Haw Yang ( $\square$ hawyang@princeton.edu ) \\ Princeton University \\ Joshua Shaevitz \\ Princeton University
}

\section{Research Article}

Keywords:

Posted Date: February 7th, 2022

DOI: https://doi.org/10.21203/rs.3.rs-1322745/v1

License: (9) This work is licensed under a Creative Commons Attribution 4.0 International License. Read Full License 


\title{
Multicolor multifocal 3D microscopy using in-situ optimization of a spatial light modulator
}

\author{
M. Junaid Amin ${ }^{1,2,3}$, Tian Zhao ${ }^{1}$, Haw Yang ${ }^{1, *}$, and Joshua W. Shaevitz ${ }^{2,3, *}$ \\ ${ }^{1}$ Department of Chemistry, Princeton University, Princeton, New Jersey 08544, USA \\ ${ }^{2}$ Department of Physics, Princeton University, Princeton, New Jersey 08544, USA \\ ${ }^{3}$ Lewis-Sigler Institute for Integrative Genomics, Princeton University, Princeton, New Jersey 08544,USA \\ *Corresponding authors: hawyang@princeton.edu, shaevitz@princeton.edu
}

\begin{abstract}
Multifocal microscopy enables high speed three-dimensional (3D) volume imaging by using a multifocal grating in the emission path. This grating is typically designed to afford a uniform illumination of multifocal subimages for a single emission wavelength. Using the same grating for multicolor imaging results in non-uniform subimage intensities in emission wavelengths for which the grating is not designed. This has restricted multifocal microscopy applications for samples having multicolored fluorophores. In this paper, we present a multicolor multifocal microscope implementation which uses the same grating optics to realize near-uniform multifocal subimage intensities across multiple wavelength emission bands. Using real-time control of an in-situoptimized spatial light modulator (SLM) implemented as a multifocal grating, we demonstrate multicolor multifocal 3D imaging over three emission bands by imaging multicolored particles as well as Escherichia coli ( $E$. coli) interacting with human liver cancer cells, at $\sim 2.5$ multicolor 3D volumes per second acquisition speed. Our multicolor multifocal method is adaptable across SLM hardware, emission wavelength band locations and number of emission bands, making it particularly suited for researchers investigating fast processes occurring across a volume where multiple species are involved.
\end{abstract}

\section{Introduction}

Multifocal microscopy allows simultaneous 3D volume imaging at a diffraction limited resolution ${ }^{1-3}$. This widefield imaging modality typically uses a diffraction grating, known as a multifocal grating, in the emission path which splits the emission light into multiple diffraction orders that are imaged as subimages side-by-side onto a camera sensor. These subimages correspond to unique object planes which are separated in object space by a distance $\Delta z$. This method allows volume imaging speeds reaching hundreds of frames per second, limited only by the camera, which makes this technique attractive for many researchers.

Although single emission wavelength multifocal imaging has been demonstrated ${ }^{1,2}$, extending multifocal microscopy to multicolor imaging applications has been challenging thus far due to the characteristic nature of the multifocal grating. The diffractive pattern on this grating is typically designed to provide uniform subimage intensities for a single emission wavelength $(\lambda)$ band having a central emission wavelength $\lambda_{\mathrm{c}}$. When these gratings are used for imaging samples having multicolored species, they lead to non-uniform subimage intensities in wavelengths for which the grating is not designed ${ }^{4,5}$. An example of this issue is shown in Fig. 1 for an SLM-based multifocal microscope optimized for $\lambda_{\mathrm{c}}=671 \mathrm{~nm}$, where a metric $M$ is used to quantify the intensity uniformity (see Section2 for metric description).

One approach to dealing with this issue is to use the same grating pattern for imaging via multiple emission bands and implement post-processing intensity scaling in the resulting non-uniformly illuminated subimages obtained in the "off-design" emission band ${ }^{4,5}$. Low-intensity subimages lead to poor signal-to-noise performances and loss of information, however. Another approach involves designing a pattern for custom-fabricated gratings to afford uniform subimage intensities in two separate predetermined emission channels ${ }^{6}$. Encouraged by these prior-art advances, we envision a new-generation multicolor multifocal platform that allows uniform subimage intensities at any emission wavelength band of choice as well as any number of emission wavelengths.

In this paper, we present such a multicolor multifocal microscope platform concept which allows uniform subimage intensities across multiple tunable emission bands using the same grating optics. This is accomplished by using in-situ optimization of an SLM deployed as the multifocal grating ${ }^{3}$ and generalizing the framework for an in-situ multicolor implementation. In principle, our approach is adaptable to any $\lambda_{c}$ and number of emission channels. These characteristics make this multicolor multifocal microscope a useful tool for various imaging applications involving multicolor species. In the rest of the paper, we first describe the optical setup, followed by a description of single-color multifocal microscopy. We then describe our approach towards multicolor multifocal operations, before presenting the experimental demonstrations. This is followed by a discussion 

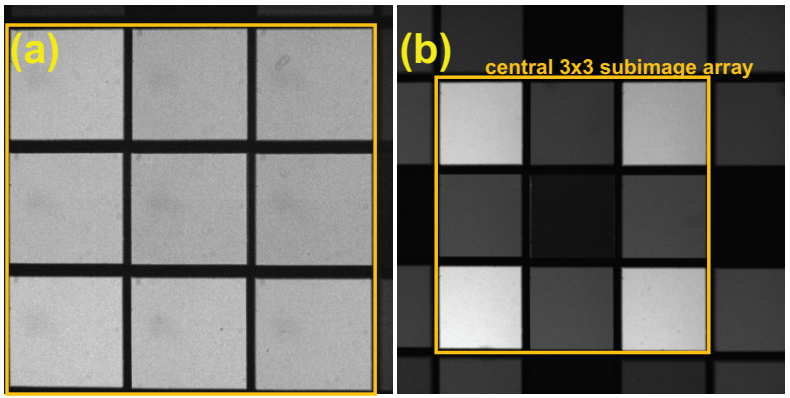

Figure 1. Example images showing the effect deploying an SLM-displayed multifocal grating pattern for an emission filter for which the pattern is not designed for. Here, an SLM pattern is displayed which was optimized for a $3 \times 3$ subimage array using an emission filter having $\lambda_{c}=671 \mathrm{~nm}$ and SLM calibration settings giving $0-2 \pi$ relative phase control for $\lambda=696 \mathrm{~nm}$. Using this pattern, corresponding bright-field mode multifocal images are obtained using emission filters having (a) $\lambda_{c}=671 \mathrm{~nm}$, which is the pattern design wavelength giving $M=0.81$, and (b) $\lambda_{c}=512 \mathrm{~nm}$, which is far from the design wavelength giving $M=0.07$. The orange rectangle indicates the camera region occupied by the central $3 \times 3$ subimage array.

section before a brief conclusion paragraph which summarizes the work.

\section{Multicolor multifocal microscope}

\section{Optical diagram}

The multicolor multifocal microscope design is shown in Fig. 2. Excitation light from multiple laser sources (three lasers are shown in Fig. 2) are directed through dichroic optics towards a sample via a microscope objective. Lens 1 focuses the excitation light onto the back focal plane of the objective to illuminate the full sample imaging field of view. Emission light from fluorophores in the sample is collected using the same objective and reflected off Dichroic 3 towards Lens 2 which forms an image at the Rectangular Aperture plane. This Rectangular Aperture limits the imaging field of view to prevent multifocal subimages from overlapping. The emission light is then directed onto the SLM placed at the Fourier plane via Lens 3. A linear polarizer is placed in the emission path to ensure phase-only operation of the SLM. A grayscale grating pattern displayed on the reflective SLM results in multiple diffraction orders of the emission light which are imaged onto a camera by Lens 4 through a multi-bandpass emission filter. The inset in Fig. 2 shows the camera placement of the subimages formed, relative to the $z=0$ plane, which are conjugate to unique object planes separated by $\Delta z$. Optional brightfield/darkfield illumination (not shown in Fig. 2) can be incorporated in the optical design for additional multifocal modalities.

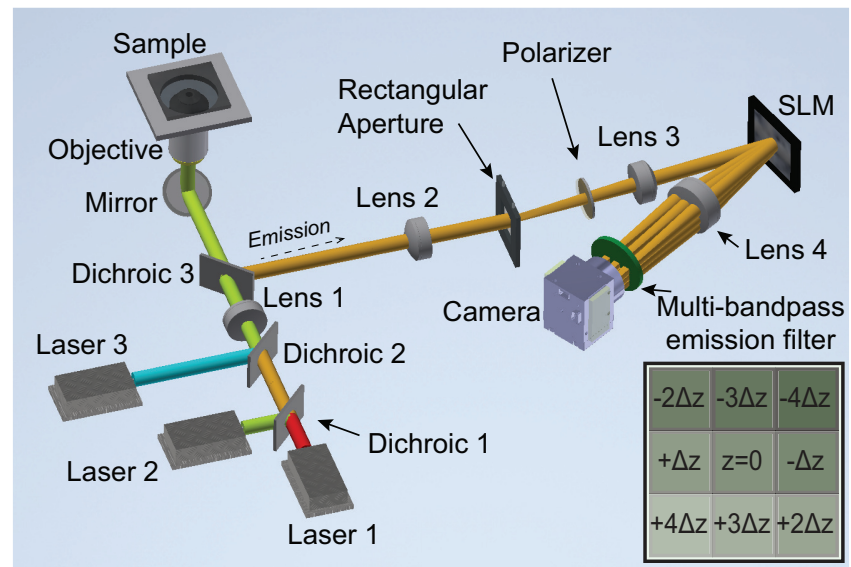

Figure 2. Optical diagram of the multicolor multifocal microscope. Inset shows the conjugate object plane arrangement for a $3 \times 3$ subimage array imaged on a camera.

\section{Multifocal imaging over a single-wavelength emission band}

Before presenting the multicolor multifocal pipeline, we first describe conventional single-wavelength emission band multifocal imaging. To begin, using the in-situ iterative algorithm ${ }^{3}$, an optimized SLM multifocal grating pattern specific to the emission 
band is acquired. Briefly, the SLM is calibrated using the calibration settings specific to the emission wavelength $\lambda_{\mathrm{c}}$ to achieve $0-2 \pi$ relative phase control corresponding to 0-255 grayscale patterns. The optimized pattern is displayed on the SLM and can readily be edited to vary $\Delta z$ without affecting the subimage intensities ${ }^{3}$. Once a desired $\Delta z$ is chosen, the pattern is displayed on the SLM and is kept unchanged throughout an imaging experiment until it finishes or a different $\Delta z$ is desired.

\section{Multifocal imaging across multiple emission bands}

By and large, there are two steps involved in generalizing multifocal imaging for arbitrary multiple emission bands using an SLM-based technology. One is the calibration of the input control voltage to the phase of each SLM pixel whereas the other is the generation of SLM-generated grating pattern for multifocal image projection. In our implementation, the former is accomplished through a separate control experiment and the latter is carried out in situ. To begin, optimized SLM patterns are obtained separately for each of the emission bands. For multicolor multifocal imaging, the same SLM calibration settings are used for operations across multiple emission bands. This is possible because of the adaptability the in-situ iterative algorithm ${ }^{3}$ which provides grating patterns giving near-uniform subimage intensities regardless of SLM calibration settings.

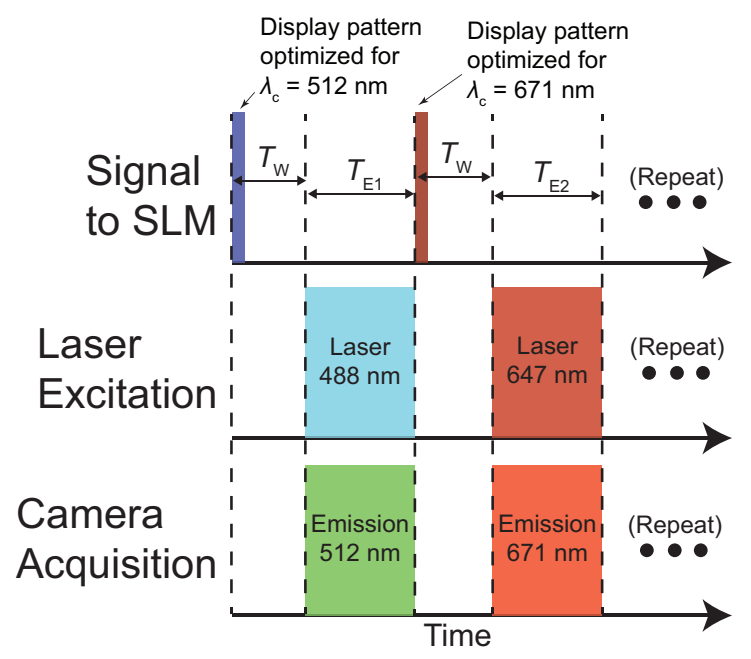

Figure 3. Example timing diagram for a two-color multifocal microscope.

Once optimized SLM patterns are obtained for the different emission bands using the same SLM calibration settings, these patterns are stored and synchronously displayed on the SLM with a multicolor excitation and image acquisition routine. A timing diagram of an example two-colored multifocal microscope illustrating this concept is shown in Fig. 3. In Fig. 3, the two-colored timing diagram assumes excitation lasers of wavelength $488 \mathrm{~nm}$ and $647 \mathrm{~nm}$, while a multi-bandpass emission filter has emission bands located at $\lambda_{\mathrm{c}}$ values of $512 \mathrm{~nm}$ and $671 \mathrm{~nm}$. To begin the multicolor multifocal imaging routine, the SLM pattern optimized for $\lambda_{\mathrm{c}}=512 \mathrm{~nm}$ is first sent to the SLM for display. A wait period of time $T_{\mathrm{W}}$ is subsequently needed to account for the $\sim 16.67 \mathrm{~ms}(60 \mathrm{~Hz})$ addressing rate of typical SLMs as well as the response time of the liquid crystals in the $\mathrm{SLM}^{7}$. After $T_{\mathrm{W}}$ has passed, the $488 \mathrm{~nm}$ laser is turned on while the camera acquires an image having an exposure time $T_{\mathrm{E} 1}$. Once the image corresponding to $488 \mathrm{~nm}$ excitation is acquired, the $671 \mathrm{~nm}$ centered emission band optimized grayscale is sent to the SLM. A wait time of $T_{\mathrm{W}}$ passes before the $647 \mathrm{~nm}$ laser turns on and the camera acquires an image of exposure time $T_{\mathrm{E} 2}$ corresponding to the emission band centered at $671 \mathrm{~nm}$. This cycle is repeated until the desired number of images are acquired. Note that this sequence can accommodate additional wavelength emission bands, each having a corresponding wait time $T_{\mathrm{W}}$ as well as acquisition time. These features make this method universally adaptable for a large variety of imaging requirements. This concludes the multicolor multifocal pipeline.

\section{Multifocal image uniformity metric $M$}

For a measured subimage intensities $\left\{I_{\mathrm{m}, \mathrm{i}}\right\}$, with $i=\{1,2, \ldots \mathrm{N}\}$ where $N$ is the number of subimages, the metric $M$ is used to quantify multifocal image intensity uniformity ${ }^{3}$ :

$$
M=\frac{\min \left(\left\{I_{\mathrm{m}, \mathrm{i}}\right\}\right)-I_{\mathrm{b}}}{\max \left(\left\{I_{\mathrm{m}, \mathrm{i}}\right\}\right)-I_{\mathrm{b}}}
$$

where $I_{\mathrm{b}}$ is a measured background intensity. The higher the subimage intensity uniformity, the higher the $M . M=1$ indicates that all subimages have equal intensities. 


\section{Results}

The multicolor multifocal microscope is implemented on a home-built aluminum block as the microscope base and controlled using an in-house developed LabVIEW ${ }^{8}$ code. The SLM used is Holoeye Pluto-VIS-056 having a pixel size of $8 \mu \mathrm{m}$, which is calibrated using a manufacturer-provided file to give $2.3 \pi$ relative phase control at $532 \mathrm{~nm}$. This is meant to provide a linear $2 \pi$ relative-phase control range for a wavelength of $611.8 \mathrm{~nm}$ using 8-bit grayscale patterns. We have empirically chosen this particular setting because it is near the mid-point of the emission band $\lambda_{\mathrm{c}}$ 's used in the experiments. These SLM calibration settings were kept fixed throughout the duration of the experiments, an important step of the multicolor multifocal design. The $\Delta_{z}$ is set to $500 \mathrm{~nm}$, and a grating period of $4 \times 4 \mathrm{SLM}$ pixels is used. The remaining experimental components used in the setup are discussed in Methods. Furthermore, details describing how $\Delta_{z}$ is controlled can be found in previous works ${ }^{3,4}$.

Using brightfield illumination, optimal SLM patterns providing near-uniform intensities across multifocal subimages are obtained using the in-situ iterative algorithm ${ }^{3,9}$ for $\lambda_{\mathrm{c}}$ values $512 \mathrm{~nm}, 610 \mathrm{~nm}$ and $671 \mathrm{~nm}$. The corresponding brightfield-mode multifocal images are displayed in Fig. 4, exhibiting high $M$ values and showing the near-uniform subimage intensities across the three empirically chosen emission bands. Note that in principle, the method is applicable for any $\lambda_{\mathrm{c}}$ location in the wavelength spectrum. The following experiments were performed following the Fig. 3 sequence, with $T_{\mathrm{W}}$ set to 13 ms. This $T_{\mathrm{W}}$ value was empirically found to afford high-performance multicolor imaging with our existing hardware, given the $16.67 \mathrm{~ms}$ addressing time and the $>70 \mathrm{~ms}$ response time (10\% to 90\%) quoted in the SLM manual provided by the manufacturer.
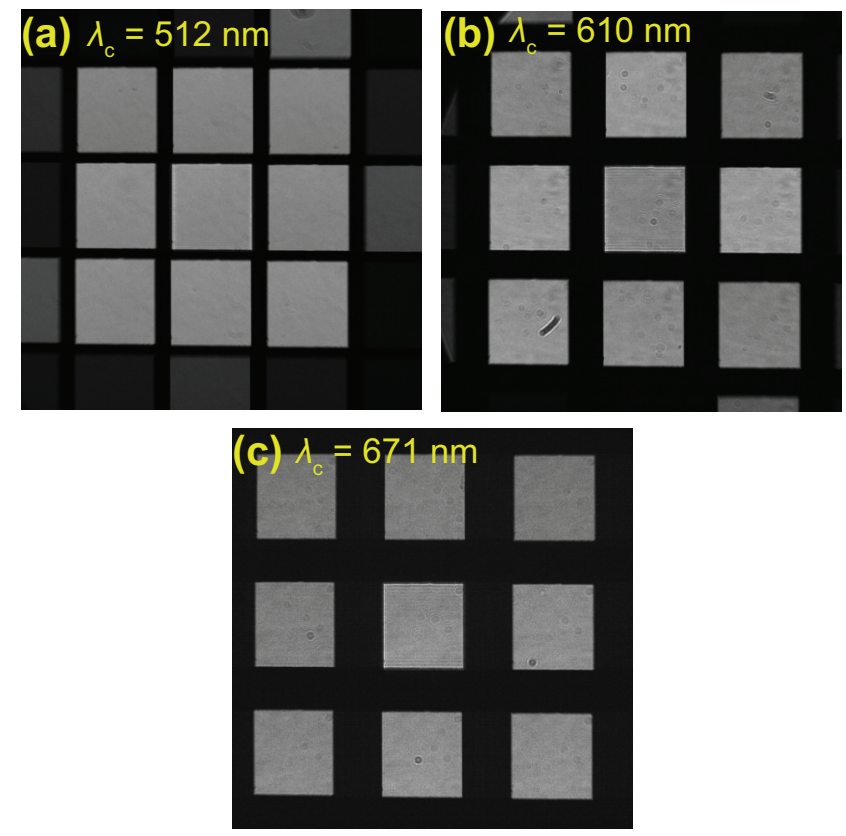

Figure 4. Using the same SLM calibration settings, shown are brightfield-mode multifocal images obtained using optimized patterns corresponding to (a) $\lambda_{\mathrm{c}}=512 \mathrm{~nm}$ with $M=0.91$, (b) $\lambda_{\mathrm{c}}=610 \mathrm{~nm}$ with $M=0.81$ (c) $\lambda_{\mathrm{c}}=685 \mathrm{~nm}$ with $M=0.79$.

Using the obtained optimized SLM patterns, we first demonstrate multicolor multifocal imaging of two-colored $100 \mathrm{~nm}$ diameter particles freely diffusing in solution. A diluted mixture of yellow-green (505/515) and red (580/605) $\mu \mathrm{m}$-sized particles (FluoroSphere, ThermoFisher Scientific) is sandwiched between two coverslips (ThermoFisher), and imaged using the microscope (see Methods for detailed sample preparation). The different colored particles were imaged using the sequence shown in Fig. 3 and the SLM patterns optimized for $512 \mathrm{~nm}$ and $610 \mathrm{~nm}$, which were illuminated by Laser 3 and Laser 2, respectively (cf. Fig. 2). Both $T_{\mathrm{E} 1}$ and $T_{\mathrm{E} 2}$ were set to $50 \mathrm{~ms}$. A single cycle of multicolor 9-plane multifocal image took $\sim 400 \mathrm{~ms}$, which includes acquisition of both color channels. Supplementary video 1 shows an image acquisition sequence of only the $512 \mathrm{~nm}$ channel at $3 \times$ real-time speed, while the $605 \mathrm{~nm}$ channel image sequence from the same dataset is shown in Supplementary video 2. Individual frames from both channels are shown in Figs. 5a and 5b. Supplementary video 3 shows the combined volume rendering, using in-house code written in $\mathrm{MATLAB}^{10}$, of the multicolored multi-particle dataset with the $512 \mathrm{~nm}$ channel shown as green and the $605 \mathrm{~nm}$ channel shown as red. A snapshot from the 3D rendered (Supplementary video 3) movie is shown in Fig. 5c. This 2.5 volumes per second multicolored particle imaging demonstration shows the utility of our system for potentially tracking multiple types of species moving over a large volume.

Next, we imaged a sample containing the bacteria $E$. coli and human liver cells (see Methods for sample preparation details) using the multicolor multifocal imaging platform. Fluorescent $E$. coli excited by Laser 3 were imaged in the $512 \mathrm{~nm}$ channel 

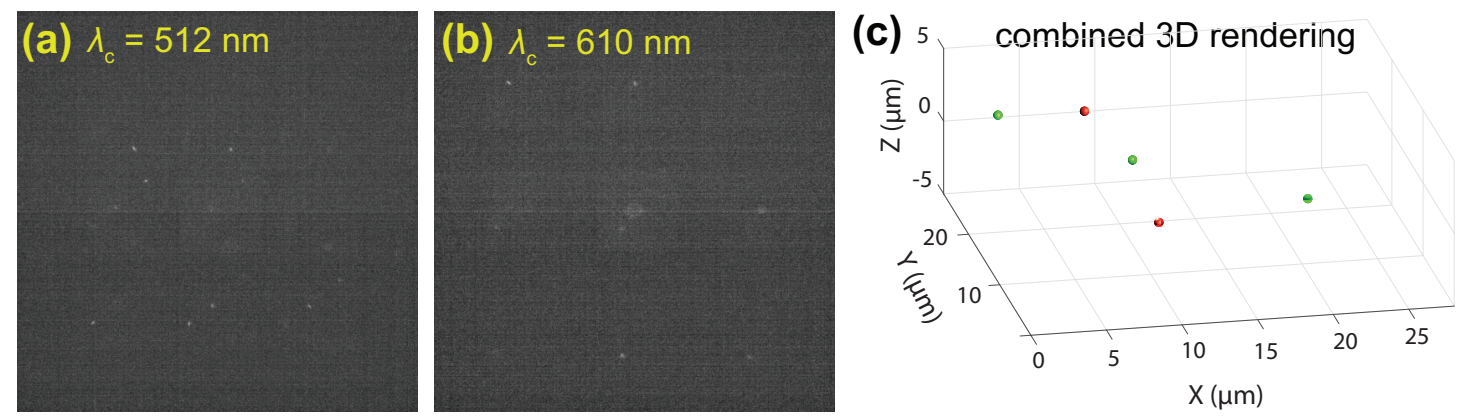

Figure 5. Example multifocal images from a sequence of two-color imaging of a mixture made up of yellow-green (505/515) and red (580/605) $\mu \mathrm{m}$-sized fluorescent particles. (a) $\lambda_{\mathrm{c}}=512 \mathrm{~nm}$ channel, and (b) $\lambda_{\mathrm{c}}=610 \mathrm{~nm}$, (c) 3D rendering of the combined multicolor multifocal images (a) and (b) showing the $\lambda_{\mathrm{c}}=512 \mathrm{~nm}$ channel particles in green and $\lambda_{\mathrm{c}}=610 \mathrm{~nm}$ in red.
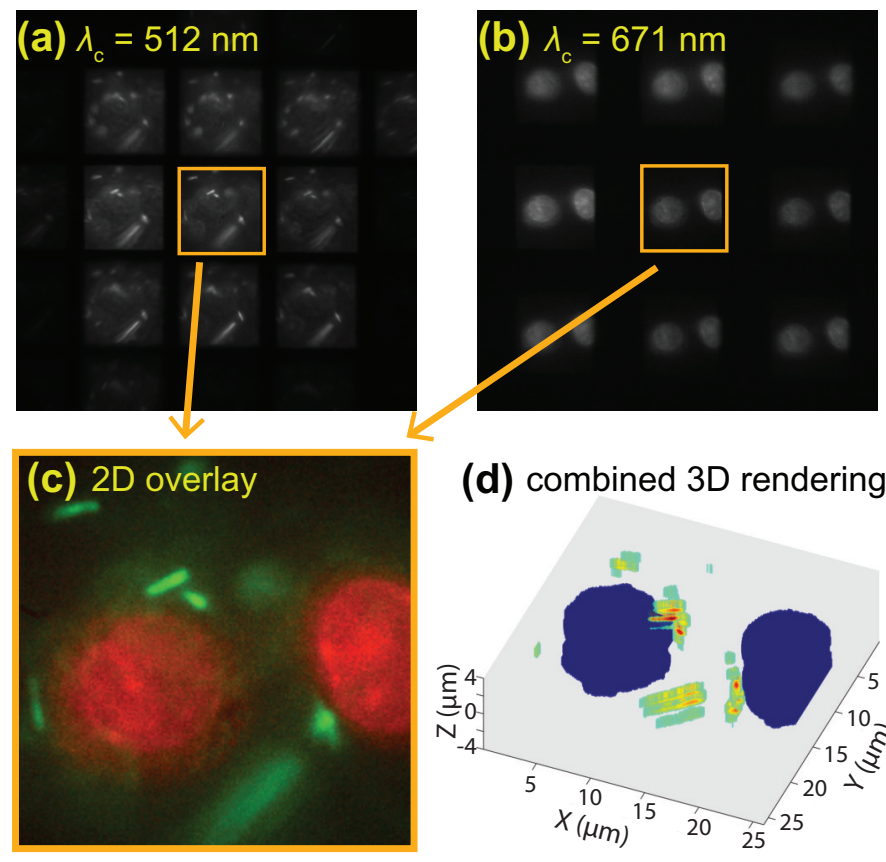

(d) combined 3D rendering

Figure 6. Example multifocal images from a sequence of two-color imaging of a sample consisting E. coli and human liver cells. (a) $\lambda_{\mathrm{c}}=512 \mathrm{~nm}$ channel, and (b) $\lambda_{\mathrm{c}}=671 \mathrm{~nm}$, (c) overlay of the central subimages corresponding to $z=0$ plane from both (a) and (b). The E. coli is colored green while the human liver cells are colored red, (d) 3D rendering of the images in (a) and (b) showing the mammalian cells (blue) and the E. coli cells (red/yellow/green).

while human liver cells were excited by Laser 1 and imaged in the $671 \mathrm{~nm}$ emission channel. Both $T_{\mathrm{E} 1}$ and $T_{\mathrm{E} 2}$ were set to $100 \mathrm{~ms}$ for this demonstration. Supplementary video 4 shows the 9-plane multifocal images of E. coli, while Supplementary video 5 displays the acquisition of human liver cells from the same multicolor dataset. Selected frames from the multicolor data acquisition are shown in Fig. 6. We also overlayed the central subimages (corresponding to only the $z=0$ plane) from both channels to show the spatial relationship of the two species (Fig. 6c and Supplementary video 6), where the E. coli is colored green and the human liver cells are colored red. In particular, Supplementary video 6 shows the E. coli pushing and pulling on the human liver cells. Note that the remaining 8 planes from each channel were unused in Fig. $6 \mathrm{c}$ as part of the overlay process, showing the large volume of information available to visualize and observe such dynamics. Fig. 6d shows a snapshot of a 3D rendered movie (Supplementary video 7) computed using the combined multicolor multifocal data as input to an in-house MATLAB rendering code. The large blue structures in Fig. 6d represent the human liver cells while the green/yellow/red structures around them represent the E. coli. The ability to acquire 3D volumetric multicolor data such as shown in Fig. 6 makes this technique suitable for investigating inter-species dynamics across a variety of systems. 


\section{Discussion}

The multicolor multifocal imaging routine presented here overcomes prior-art issues of non-uniform subimage intensities during multicolor image acquisitions. Our in-situ optimization method for SLM as the multifocal grating, which allows high performance multicolor multifocal imaging, is adaptable across multiple microscope types and SLM hardware manufacturers, allowing access to many researchers. Our design allows the use of the same SLM calibration settings for different wavelengths of operations. Empirically, the exact choice of the $0-2 \pi$ calibration wavelength is not important as long as the calibration wavelength for $2 \pi$ relative phase control is near the desired emission $\lambda_{\mathrm{c}}$ values. Also, note that during the grating-pattern optimization process for each emission wavelength in an in-situ application, other emission bands are spectrally blocked to prevent algorithm failure due different emission bands being illuminated simultaneously.

The multicolor imaging speeds depend significantly on the hardware used. In our current setup, we used $T_{\mathrm{W}}=135 \mathrm{~ms}$. This wait time was obtained experimentally by measuring how long it took for a new SLM grating pattern to stabilize on the camera by evaluating the resulting $M$ values. Different SLMs have different response times which depends on a range of factors including the liquid crystal pixel characteristics as well as the drive voltage settings. Deploying SLMs with faster response times will increase the volume imaging speed of the proposed multicolor multifocal routine. Furthermore, note that the location of subimages in the multifocal images changes shown for different $\lambda_{\mathrm{c}}$ values as expected according to the grating equation ${ }^{11}$.

Finally, the large volumes of sample information provided by the multicolor multifocal microscope as well as their variation across camera regions due to different emission wavelength bands indicate a need for specialized post-processing techniques. Although researchers have implemented various reconstruction methods for multifocal imaging volumes ${ }^{12,13}$, the extension to multicolor imaging presents unique computational challenges yet opportunities for future research, such as deciphering 3D shapes from multiple focal plane images of different shaped objects interacting with each other (e.g., Fig. 6).

\section{Conclusion}

In conclusion, a multicolor multifocal microscopy platform is presented. Using in-situ optimization of an SLM used as the multifocal grating, we show that it is possible to provide near-uniform multifocal subimage intensities for three emission wavelength bands in the visible range. Using this idea, as demonstrated by our platform, researchers can optimize multifocal experiments according to desired dye emission wavelengths as well as the number of emission channels. This promises to be a useful tool for researchers probing volume dynamics across a variety of biological and material systems.

\section{Methods}

\section{Optical setup components}

The components used in the experimental setup are: Laser 1: Cobolt 06-01 series with wavelength of $647 \mathrm{~nm}$ and maximum power of $130 \mathrm{~mW}$, Laser 2: Cobolt 08-01 series with wavelength of $561 \mathrm{~nm}$ and maximum power of $100 \mathrm{~mW}$, Laser 3: Cobolt 06-01 series with wavelength of $488 \mathrm{~nm}$ and maximum power of $120 \mathrm{~mW}$, Dichroic 1: Semrock Di03-R561-t3-25x36, Dichroic 2: Semrock's Di03-R488-t3-25x36, Dichroic 3: Semrock's Di03-R405/488/561/635-t3-25x36, Microscope objective: Leica HC PL APO 100x magnification with Numerical Aperture adjustable between 0.7 to 1.4, focal length of Lens 1: $150 \mathrm{~mm}$, focal length of Lens 2, Lens 3 and Lens 4: 200 mm, Rectangular Aperture from Ealing (Hyland Optical Technologies), Linear Polizer: model LPVISE100-A from Thorlabs, Camera: Orca Flash 4 V3 from Hamamatsu. The Multi-bandpass filter deployed was custom-made by Alluxa having the central wavelengths ltocated at $460 \mathrm{~nm}, 512 \mathrm{~nm}, 610 \mathrm{~nm}$ and $671 \mathrm{~nm}$, with corresponding bandwidths of $21 \mathrm{~nm}, 15 \mathrm{~nm}, 16 \mathrm{~nm}$ and $20 \mathrm{~nm}$, respectively. The Rectangular Aperture was controlled such that the total field of view per subimage was set to $\sim 27 \mu \mathrm{m}^{2}$.

\section{Multi-particle sample preparation}

Yellow-green fluorescent (505/515) carboxylate-modified FluoSpheres of $0.1 \mu \mathrm{m}$ and Red (580/605) carboxylate-modified FluoSpheres of $0.1 \mu \mathrm{m}$ were purchased from ThermoFisher Scientific. $10 \mu \mathrm{L}$ of $10^{4} \times$ diluted yellow-green particles was mixed with $20 \mu \mathrm{L}$ of $10^{4} \times$ diluted red particles. $10 \mu \mathrm{L}$ of this mixture was then added to $90 \mu \mathrm{L}$ of Thiodiethanol (SigmaAldrich). $15 \mu \mathrm{L}$ of this mixture is dropped on a $22 \mathrm{~mm} \times 22 \mathrm{~mm}$ coverslip (ThermoFisher) using a pipette. A $18 \mathrm{~mm} \times 18 \mathrm{~mm}$ coverslip was then placed on top of this droplet which was then spread throughout the top coverslip. The sample was imaged via the bottom coverslip.

\section{Bacteria/liver cell sample preparation}

A pipette tip was used to scrape a stock of frozen E. coli MG1655 KanR having the Green Fluorescent Protein (GFP) plasmid pWR20. The pipette tip was then dipped into $3 \mathrm{~mL}$ of Lysogeny Broth (LB) media in a tube. $15 \mu \mathrm{L}$ of Kanamycin was added to this mixture to neutralize the non-GFP plasmid carrying bacteria and incubated at $37^{\circ} \mathrm{C}$ for 18 hours. After the incubation 
period, $15 \mu \mathrm{L}$ of the bacteria solution was added to $50 \mu \mathrm{L}$ of the liver-cell solution (see description below). This volume was dropped on a coverslip which was then placed on the microscope stage for imaging.

HEP G2 human liver cancer cells (HB-8065) were purchased from ATCC and cultured in Eagle's Minimum Essential Medium (ATCC 30-2003) with 10\% fetal bovine serum supplement (Gibco) using Nunclon Delta dish ( $60 \mathrm{~mm} \times 15 \mathrm{~mm})$. Cells were passaged every 5-7 days at $\sim 80 \%$ confluence. SYTO 62 red nucleic acid stain was purchased from Invitrogen and used as directed. The culture medium of cells which had $\sim 80 \%$ confluence were removed and the cells were further rinsed by $1 \mathrm{~mL}$ DPBS (Dulbecco's phosphate-buffered saline without $\mathrm{Mg}^{2+}$ and $\mathrm{Ca}^{2+}$ ). $1 \mu \mathrm{L}$ of the $5 \mathrm{mM}$ SYTO 62 stock solution was added to $1 \mathrm{~mL}$ of DPBS (without $\mathrm{Mg}^{2+}$ and $\mathrm{Ca}^{2+}$ ) to make a $5 \mu \mathrm{M}$ staining solution. The cells were stained in the culturing dish by adding $1 \mathrm{~mL}$ of the staining solution. The cells were incubated for 20 minutes, followed by $1 \mathrm{~mL}$ DPBS (without $\mathrm{Mg}^{2+}$ and $\mathrm{Ca}^{2+}$ ) rinsing. The cells were then dissociated from the dish surface by adding $1 \mathrm{~mL}$ trypsin. After 10 minutes of incubation, the cells were transferred to solution phase for centrifuging for 5 minutes at $1000 \mathrm{rpm}$. The supernatant was removed and $2 \mathrm{~mL}$ DPBS (without $\mathrm{Mg}^{2+}$ and $\mathrm{Ca}^{2+}$ ) was added to remove the unbonded dye molecule residuals. This cleaning process was repeated twice. The stained HEP G2 cells were redissolved back to $2 \mathrm{~mL}$ DPBS (without $\mathrm{Mg}^{2+}$ and $\mathrm{Ca}^{2+}$ ) for multicolor multifocal experiments.

\section{References}

1. Dalgarno, P. A. et al. Multiplane imaging and three dimensional nanoscale particle tracking in biological microscopy. Opt. Express 18, 877-884 (2010).

2. Walker, B. J. \& Wheeler, R. J. High-speed multifocal plane fluorescence microscopy for three-dimensional visualisation of beating flagella. J. Cell Sci. 132, jcs231795 (2019).

3. Amin, M. J., Petry, S., Yang, H. \& Shaevitz, J. W. Uniform intensity in multifocal microscopy using a spatial light modulator. PLoS One 15, e0230217 (2020).

4. Abrahamsson, S. et al. Fast multicolor $3 \mathrm{~d}$ imaging using aberration-corrected multifocus microscopy. Nat. Methods 10, 60 (2013).

5. Hajj, B. et al. Whole-cell, multicolor superresolution imaging using volumetric multifocus microscopy. Proc. Natl. Acad. Sci. U.S.A. 111, 17480-17485 (2014).

6. Hajj, B., Oudjedi, L., Fiche, J.-B., Dahan, M. \& Nollmann, M. Highly efficient multicolor multifocus microscopy by optimal design of diffraction binary gratings. Sci. Rep. 7, 5248 (2017).

7. Zhang, Z., You, Z. \& Chu, D. Fundamentals of phase-only liquid crystal on silicon (lcos) devices. Light. Sci. \& Appl. 3, e213-e213 (2014).

8. Bitter, R., Mohiuddin, T. \& Nawrocki, M. LabVIEW: Advanced programming techniques (CRC Press, 2006).

9. Amin, M. J., Petry, S., Yang, H. \& Shaevitz, J. W. Intensity uniformity optimization in spatial-light-modulator-based multifocal microscope. In Three-Dimensional and Multidimensional Microscopy: Image Acquisition and Processing XXVIII, vol. 11649, 116490P (International Society for Optics and Photonics, 2021).

10. MATLAB. version 7.10.0 (R2020b) (The MathWorks Inc., Natick, Massachusetts, 2020).

11. Born, M. \& Wolf, E. Principles of optics: electromagnetic theory of propagation, interference and diffraction of light (Elsevier, 2013).

12. Tian, Y. et al. Improved three-dimensional reconstruction algorithm from a multifocus microscopic image sequence based on a nonsubsampled wavelet transform. Appl. Opt. 57, 3864-3872 (2018).

13. Yoo, S. et al. 3d image reconstruction from multi-focus microscope: axial super-resolution and multiple-frame processing. In 2018 IEEE International Conference on Acoustics, Speech and Signal Processing (ICASSP), 1453-1457 (IEEE, 2018).

\section{Acknowledgements}

This work was supported by Princeton University Eric and Wendy Schmidt Transformative Technology Fund (to JWS and HY) and the U.S. Department of Energy (DE-SC0019364, to HY).

\section{Author contributions statement}

M.J.A. conducted the experiment, collected, analyzed the data, wrote the original draft, and revised the manuscript. T.Z. prepared the biological samples and revised the manuscript. H.Y. and J.W.S. designed and supervised the experiment and revised the manuscript. All authors have read and approved of the final manuscript. 


\section{Competing interests}

The authors declare no competing interests.

\section{Data Availability Statement}

The data used to support the findings of this study are available from the corresponding author upon request. 


\section{Supplementary Files}

This is a list of supplementary files associated with this preprint. Click to download.

- Suppvid1100nmparticle512nm6fps.avi

- Suppvid2100nmparticle610nm6fps.avi

- Suppvid3multiparticlereconstructionDCIMG0002.avi

- Suppvid4Ecoli512nmemission.avi

- Suppvid5LiverCells671nmemission.avi

- Suppvid6centralorderONLYEcoliLiverCells.avi

- Suppvid7RenderingEColimammalianCell.avi 